\title{
SUBORDINATE CLAUSES AND INTERRUPTION CLAUSES
}

\author{
Maynard M. Eyestone \\ University of Michigan
}

ENGLISH displays a series of clauses whose functions represent gradual steps from subordination to complete lack of grammatical relationship. Consider the following examples:

1. How did you do it? I did it as I always have at this time of year.

2a. I did it, as I always have at this time of year, with pruning shears.

b. I did it, as you know, with pruning shears.

3a. I did it, you know, with pruning shears.

b. They do it, let's say, with pruning shears.

4a. They do it, let's say with pruning shears, starting about December first.

b. They do it with, let's say pruning shears, starting about December first.

5. They prune their trees, and I think with reason, starting about December first.

6. They prune their trees every winter-and it's a difficult jobin order to get the best possible crop the following year.

7. They prune their trees every winter-it's necessary in order to get a good crop the following year-using pruning shears and pruning saws.

Let us refer to the underlined clauses by number: $1,2 \mathrm{a}$, $2 b$, etc., and the types they represent as types $1,2 a$, etc. We may also identify clauses $2 \mathrm{a}$ and $2 \mathrm{~b}$ as representing a single "type $2, "$ clauses $3 \mathrm{a}$ and $3 \mathrm{~b}$ as "type 3 ," etc. 
The subordinate clause may be defined as a structure of predication (a finite verb with its subject, or an imperative verb without a subject) functioning as substantive or modifier. Clause 1 is a subordinate clause modifying the predicate did it.

Clauses $3 \mathrm{a}$ and $3 \mathrm{~b}$ are like those described by Nida ${ }^{l}$ as "Attributives to Clauses: Parenthetical: These . . . expressions are only associated with the clause formally. (They) are part of the sentence phonologically, and are dependent upon it, in that they do not constitute complete linguistic utterances. (They) do not pattern in any endocentric or exocentric pattern." The latter statement means that they neither attach themselves to another structure as modifier nor do they function as a substantive.

In contrast with our clause 1 , clauses $3 a$ and $3 b$ appear at first glance to be not in modifying function, and therefore not subordinate clauses. Nida's "parenthesis" would appear to be something other than a "subordinate clause." Parentheses, evidently, are recognizable as set off by pause from the clause with which they are "associated formally." In clauses 1 and 3 there is also the difference of the presence or absence of a subordinate conjunction such as as; but of course many subordinate clauses have a zero marker (". . . the trees you pruned this morning. . .") and the presence of an audible marker cannot be made a requisite of membership in the subordinate clause class. It is to be observed that clauses $3 \mathrm{a}$ and $\mathbf{3 b}$ are incomplete, or in Nida's terms, "dependent" upon the clause in which they are contained. Thus pause-separation and dependence appear to be chief characteristics of the "parenthesis."

Pause separation and independence characterize clause 7 . It might function just as it stands (even without change of suprasegmentals) as a separate sentence. Clause 7 is not a "parenthesis." It is an "inter ruption." An inter ruption occurs between two parts of a single grammatical sequence and is not grammatically dependent on the clause associated formally with it.

Clauses of types 1,3 , and 7 seem to represent three syntactic categories which we might name subordinate clause, parenthesis, and interruption. But to what category do the other examples belong? And do they shed light on whether our three classes might be reduced to two?

Let us look at $2 \mathrm{a}$ and $2 \mathrm{~b}$. Here, as in 1 , the conjunction as is present; and as in $3 \mathrm{a}$ and $3 \mathrm{~b}$, pause-separation is present. $\widetilde{\text { It }}$ is quite clear (though I can state no evidence beyond the intuition of native speakers) that the structural meaning of $2 \mathrm{a}$ and

\footnotetext{
${ }^{I}$ Eugene A. Nida, A Synopsis of English Syntax, Doctoral dissettation, University of Michigan, 1943. Ed. Benjamin Elson (Norman, Oklahoma: SIL of the U. of Okla., 1960) pages 188-18\%.
} 
$2 b$ is the same as that of $3 a$ and $3 b$. Pause-separation and dependence, the chief characteristics of the parenthesis, are present in clauses of type 2 . We conclude that these are parentheses. We now have marked and unmarked parentheses.

If clauses of types 2 and 3 are members of the same syntactic class, we must call our parentheses a class of subordinate clauses. It seems clear in the case of type 2, if not in that of type 3, that the parenthetical clause is a clause-modifier, a kind of clause adverbial-what Nida calls a "sentence adverb expression" and others have called a "sentence adverbial." Compare $2 a$ with "I did it, predictably, with pruning shears."

An utterance like the following might occur: "I did it, as I have always done it at this time of year, with pruning shears." (Compare 2a.) The clause set off by pauses in this case is independent, except for the "non-independence" imposed on it by the conjunction as; it is not dependent on the containing clause. We might therefore suggest that this clause is more like type 7 than like type 2. But like 2a and $2 b$, this clause is in clausemodifying function. We will therefore want to say that the parenthesis, or clause-modifying clause, is characterized by either dependence or the presence of a subordinate conjunction.

Clauses $4 a$ and $4 b$ differ from clauses of type 3 in only one way: Part of that on which the parenthesized structure of predication is dependent is included between the pauses. In looking at $4 \mathrm{a}$, we might be tempted to say that this is a variation of the "interruption" (clause 7), with zero substitution of they do it. It would be highly arbitrary, of course, to single out interruptions and say that zero substitution could not occur in that particular structure; and assuming the substitution of zero for they do it, the clause "let's say with pruning shears" meets the requirements of membership in the "interruption" class: pause-separation and independence.

But essentially the same reason could be advanced for inclusion of $3 \mathrm{a}$ and $3 \mathrm{~b}$ (and except for the conjunction, $2 \mathrm{a}$ and $2 \mathrm{~b}$ ) in the interruption class; and we cannot deny the sentenceadverbial function of clauses of types 2 and 3 . They are modifiers, and not interruptions. Clauses $4 \mathrm{a}$ and $4 \mathrm{~b}$ must be modifiers, too.

We therefore include $4 \mathrm{a}$ and $4 \mathrm{~b}$ in our class of parentheses or clause-modifying clauses.

In $4 \mathrm{~b}$ we see two-way dependence. The parenthesis is dependent on the containing clause, and the containing clause is dependent on the parenthesis. The dependence of let's say with pruning shears puts it in the clause-modifying class. The dependence of the containing clause is incidental. (We can find 
examples of our "interruption" class, also, in which the container is dependent. In both classes, container dependence is unusual.)

So we say that clauses of types 2,3 , and 4 are clausemodifying subordinate clauses, characterized by pause-separation and either subordinate conjunctions or dependence on the clause with which they are formally associated.

Let us now consider clause 6 . Like 7 , it has pause-separation and independence. It is an "interruption." It differs from 6 in being introduced by a coordinate conjunction (one which occurs not only between pairs of clauses but also between pairs of other linguistic forms.) The conjunction hints at a closer relationship with the container than is evidenced in 7 . There is a relationship here of coordination, but the speaker has chosen to structure his remarks in such a way that the coordination is hinted at rather than made central.

Clauses such as 5 occur, from which independence, a chief sign of the interruption clause, is absent. Like 6, 5 is introduced by a coordinate conjunction, but here, as in $4 \mathrm{a}$ and $4 \mathrm{~b}$, zero substitution is employed, and the clause is dependent on its containing clause. We may feel that 5 is more like 4 than like 6 , and it certainly represents a "gray" area. But I think we can say that its function is not modification, but interruption, and that it doesn't qualify, but merely adds information. If we include clauses like 5 in the interruption class, we must say that interruption clauses are characterized by either independence or the presence of a coordinate conjunction.

To summarize: Clauses 5, 6, and 7 are "interruption clauses" characterized by (1) pause-separation, (2) independence or a coordinate conjunction marker, and (3) a function other than that of modifier. Clauses of types 2, 3, and 4 are "parentheses" or "clause-modifying subordinate clauses," characterized by (1) pause-separation, (2) dependence or a subordinate conjunction marker, and (3) the function of clause-modifier. Clause 1 is a predicate-modifying subordinate clause, characterized by lack of pause-separation and of course by certain features of word-class selection and arrangement which characterize subordinate clauses in general.

Definitions of "clause-modifying clauses" and "interruption clauses" are now possible. A clause set off by pause from a clause with which it is associated formally (which contains it or to which it is tied by supra-segmental features) and which does not function as substantive or as modifier of some structure other than a clause, is either (1) a clause-modifying clause or (2) an interruption clause. It is a clause-modifier if it either (1) is introduced by a subordinate conjunction or (2) is dependent 
for the completion of its structure on the clause with which it is associated. It is an interruption clause if it either (1) is introduced by a coordinate conjunction or (2) is independent of the clause with which it is associated. 